\title{
Genome-wide association study discovered favorable single nucleotide polymorphisms and candidate genes associated with ramet number in ramie (Boehmeria nivea L.)
}

Kunmei Chen ${ }^{*}$, Mingbao Luan ${ }^{\dagger}$, Heping Xiong, Ping Chen, Jikang Chen, Gang Gao, Kunyong Huang, Aiguo Zhu* and Chunming Yu*

\begin{abstract}
Background: Ramie (Boehmeria nivea L.) is one of the most important natural fiber crops and an important forage grass in south China. Ramet number, which is a quantitative trait controlled by multigenes, is one of the most important agronomic traits in plants because the ramet number per plant is a key component of grain yield and biomass. However, the genetic variation and genetic architecture of ramie ramet number are rarely known.

Results: A genome-wide association study was performed using a panel of 112 core germplasms and 108,888 single nucleotide polymorphisms (SNPS) detected using specific-locus amplified fragment sequencing technology. Trait-SNP association analysis detected 44 significant SNPs that were associated with ramet number at $P<0.01$. The favorable SNP Marker20170-64 emerged at least twice in the three detected stages and was validated to be associated with the ramie ramet number using genomic DNA polymerase chain reaction with an $F_{1}$ hybrid progeny population. Comparative genome analysis predicted nine candidate genes for ramet number based on Marker20170-64. Real-time quantitative polymerase chain reaction analysis indicated that six of the genes were specific to upregulation in the ramie variety with high ramet number. These results suggest that these genes could be considered as ramet number-associated candidates in ramie.

Conclusions: The identified loci or genes may be promising targets for genetic engineering and selection for modulating the ramet number in ramie. Our work improves understanding of the genetics of ramet number in ramie core germplasms and provides tools for marker-assisted selection for improvement of agricultural traits.
\end{abstract}

Keywords: Ramie, Ramet number, Genome-wide association study, Significant SNPs, qPCR

\section{Background}

Ramie (Boehmeria nivea L.), which is native to China and commonly known as China grass, is one of the oldest fiber crops worldwide [1]. It has a history of over 4000 years as a fiber crop in China and has been popularly used as animal feed and for the phytoremediation of heavy metal-contaminated farmlands over the past decades. The superior fiber obtained from its woody stem is long and highly durable, pure white in color, and

\footnotetext{
*Correspondence: chenkunmei@caas.cn; zhuaiguo@caas.cn; yuchunming@caas.cn

${ }^{\dagger}$ Kunmei Chen and Mingbao Luan contributed equally to this work. Institute of Bast Fiber Crops, Chinese Academy of Agricultural Sciences, No. 348, West Xianjiahu Road, Changsha 410205, Hunan Province, China
}

silky in texture, with a high degree of hygroscopicity and superior heat dissipation. These superior characteristics make ramie a highly versatile and useful natural raw textile material. Generally, raw ramie fiber can be preserved for 20-30 years. The peak period of global ramie production occurred between 2001 and 2007, and this provided many raw ramie stocks, that have met the processing and consumption demand in recent years. The total production of ramie worldwide has declined in recent years, whereas the consumption has increased annually (http:// www.fao.org/faostat/en/\#home), indicating an increasing requirement for raw ramie fiber. High-end textile products fabricated with natural fiber have become increasingly

(C) The Author(s). 2018 Open Access This article is distributed under the terms of the Creative Commons Attribution 4.0 International License (http://creativecommons.org/licenses/by/4.0/), which permits unrestricted use, distribution, and 
popular and, consequently, the demand for ramie raw textile material could be predicted to rise in the future. Ramie has a high tolerance and strong ability to absorb multiple heavy metals such as cadmium [2], lead [3], and arsenic [4] from contaminated soil. It is therefore considered to be one of the most potentially useful plants for remediation of heavy metal contaminated land. Plants with high biomass have an advantage in heavy metal absorption and, thus, improving the biomass of ramie is meaningful. In addition, because ramie is used as animal feed, high biomass is beneficial for promoting the development of the ramie feed industry. Taken together, these factors indicate the considerable significance of improving the fiber and biomass yield of ramie.

The number of ramet (in clonal plants), or tiller (in monocotyledons), is an important factor influencing crop yield [5]. For instance, the tillering ability is one of the most important traits in rice since it can have a significant effect on the future production of panicles [6], which in turn is highly correlated with grain yield [7]. For perennial plants, the ramet formation ability is an important indicator of their potential biomass production. In Eupatorium adenophorum, a perennial clonal plant, the biomass increases with increasing ramet number [8]. Ramie is a perennial herbaceous plant, and its ramet number is one of the most important targets in ramie breeding. Compared with the plant height and leaf number, the ramet number is the main factor affecting ramie biomass [9]. Bai et al. [10] showed that there is a significant correlation between fiber yield and ramet number in ramie. Therefore, improving the ramet number in ramie is important for the biomass or the fiber product.

Single nucleotide polymorphism (SNP) refers to the DNA sequence polymorphism caused by the variation of single nucleotide at the genomic level. SNP often occurs in the genome and is the most abundant and stable form of genetic variation, which provide valuable markers for the study of agronomic traits in crops [11]. Specific locus amplified fragment sequencing (SLAF-seq) is a relatively new high-resolution strategy that can fast, accurately, efficiently, and economically develop large-scale SNP and InDel markers. The flowchart of SLAF-seq technology comprises: i) pre-design scheme for SLAF selection, ii) SLAF-seq library construction, and iii) highthroughput sequencing and genotyping [12].

Ramet or tiller number per plant is a quantitative trait controlled by multigenes, and is reported to be equally regulated by additive and dominant gene effects [13]. Genome-wide association study (GWAS) is a powerful tool for complex trait dissection in plants [14]. Compared to biparental linkage mapping, GWAS has the advantages of high resolution, cost-efficiency, and not requiring the creation of a mapping population. With the rapid development of DNA and RNA-sequencing technologies, high-density genotyping with single nucleotide polymorphisms (SNPs) has become easily accessible, enabling GWAS to be performed in many plants including maize [15], rice [16], cotton [17], canola [18], sorghum [19], foxtail millet [20], and Arabidopsis [21].

Significant SNPs and candidate genes associated with quantitative traits have been detected in a large variety of plants using GWAS. In cotton, one favorable SNP associated with verticillium wilt resistance was identified from 17 significant SNPs detected using GWAS based on SLAF-seq technology, and 22 candidate genes for verticillium wilt resistance were predicted based on a favorable SNP [22]. Six candidate genes with pleiotropic effects on stalk cell wall components in maize have been identified using the GWAS method [23]. Furthermore, in Arabidopsis, a gene associated with leaf arsenic accumulation was identified using GWAS [24]. Previously, five QTLs were identified in an $\mathrm{F}_{2}$ agamous line population of ramie using 114 SSR markers, although they were not confirmed in a different population [25]. To better understand the genetics of ramie ramet formation, further investigations are needed.

To investigate the genetic components underlying the natural variation in ramie germplasms and discover favorable SNPs associated with ramet number, we performed a GWAS using SLAF-seq technology on 112 ramie core germplasms. The favorable SNPs associated with ramet number were further validated in a hybrid progeny population using a genome DNA polymerase chain reaction (PCR) strategy. Furthermore, the candidate genes associated with ramet number are discussed. This study will be helpful in clarifying the genetic structure of the ramie core germplasms and providing information about candidate quantitative trait loci (QTL) and genes that control the ramet number in ramie.

\section{Material and methods Ethics statement}

The collection of ramie specimens used in this study were planted in our scientific research field, which is owned by our institution. Therefore, no specific permissions were required for using these specimens.

\section{Plant materials and statistics of ramet number}

A total of 112 core germplasms of ramie planted in the Institute of Bast Fiber Crops, Chinese Academy of Agricultural Sciences were used for SNP development in this study. These germplasms were collected from China, India, Indonesia, Brazil, and Cuba (Additional file 1). An $\mathrm{F}_{1}$ hybrid progeny population and its parents (two ramie varieties: Zhongzhu NO.1 and Hejiangqingma with high and low ramet numbers, respectively), were used for SNP validation using PCR. All ramie plants were propagated by 
asexual propagation, and planted in a field ( $\mathrm{N} 28^{\circ} 21^{\prime}, \mathrm{E}$ $112^{\circ} 59^{\prime}$ ) at Changsha, China, in 2014. For each core germplasm, four plants were taken from the side branches of the same parent plant, and these were planted in a row with four plots (one plant per plot), with a distance of 40 $\mathrm{cm}$ between plots and $60 \mathrm{~cm}$ between rows. Each $F_{1}$ hybrid progeny was planted with one plant per plot, four plots per row, with $40 \mathrm{~cm}$ between plots and $60 \mathrm{~cm}$ between rows. For the 112 core germplasms and the $F_{1}$ hybrid progeny, three biological replicates were set up with a randomized block design for each replicate.

The ramet number of the core germplasm was recorded in May, August, and November 2016, when the plants had grown to $80 \mathrm{~cm}$ in height. The ramet number of the $F_{1}$ hybrid progeny population was recorded in March and November 2017.

\section{SNP genotyping and quality control}

Fresh leaves of four plants were mixed as one sample for each core germplasm. Total genomic DNA was extracted from each sample using the modified cetyltrimethylammonium bromide method described by Luan et al. [26]. DNA quality and quantity were determined using a Nanodrop 2000 spectrophotometer (Thermo Fisher Scientific, Wilmington, DE, USA) and an Agilent 2100 Bioanalyzer (Agilent Technologies, Waldbronn, Germany). Quantified DNA was diluted to $100 \mathrm{ng} \mu \mathrm{L}^{-1}$ for SLAF sequencing. The SLAF library was constructed as previously described [12] with slight modifications. The genome of Cannabis sativa (ftp://ftp.ncbi.nlm.nih.gov/genomes/all/ GCA/003/417/725/GCA_003417725.2_ASM341772v2/) was used as a reference to electronically predict the result of enzyme digestion and determine an optimal restriction enzyme solution according to the following criteria: (1) the proportion of restriction fragments in the repeated sequence is as low as possible, (2) restriction fragments are distributed as evenly as possible in the genome, (3) simulated fragments align uniquely to the reference genome, and (4) a high number of SLAF tags [27]. Furthermore, the restriction enzyme combination of RsaI and HaeIII was selected. Plant DNA was digested with a combination of RsaI + HaeIII (NEB, Ipswich, MA, USA) to obtain the SLAF tags (defined as enzyme fragment sequences of 264-394 bp), followed by dual-index paired-end adapter ligation, PCR amplification, and target fragment selection for the SLAF library construction. The selected fragments and a control (Oryza sativa ssp. japonica) were sequenced using the Illumina Hi-Seq 2500 sequencing platform (Illumina Inc., San Diego, CA, USA) at Biomarker Technologies Corporation in Beijing (http://Biomarker.com.cn/). The raw data were assessed using the dual-index to obtain reads for each sample. After filtering out adapter reads, the sequence quality was evaluated by analyzing the guanine-cytosine content and the Q30 quality score $\left(\mathrm{Q}=-10 \times \log _{10}^{\mathrm{e}}\right.$, indicating a $0.1 \%$ chance of an error and, thus, $99.9 \%$ confidence) quality score. Effectiveness and accuracy were evaluated using enzyme-cut rate information from the control. All reads were checked using cluster analysis based on sequence similarity. Reads from different samples were classed into one set named the SLAF tag. A SLAF tag that exhibited differences in sequences from other samples was defined as polymorphic. Using the Burrows-Wheeler Alignment tool software [28], the sequenced reads were compared with the reference tag, which was the deepest sequence in each SLAF tag. The GATK38 [29] and SAMtools [30] packages were used to perform SNP calling, and the SNPs obtained by both of these methods were treated as reliable SNPs. Finally, the reliable SNPs were filtered out with integrity $>0.8$ and $\mathrm{MAF}>0.05$.

\section{Population structure and kinship analysis}

The population structure of the 112 core germplasms was analyzed using an admixture software [31]. The number of simulation subgroups ( $K$ value) was set from 1 to 10 . The statistic $\Delta K$ was calculated using STRUCTURE HARVESTER [32] (http://taylor0.biology.ucla.edu/ structureHarvester/). The $\Delta K$ was set as the determinant factor for evaluating the optimal value of $K$ [33]. The Q-matrix was obtained using the CLUMPP software [34]. The phylogenetic tree of the 112 core germplasms was constructed using MEGA 5.1 with the neighborjoining (NJ) method (1000 bootstraps) [35]. The kinship (K) matrix was estimated using SPAGeDi version 1.4b [36].

\section{GWAS}

Phenotype-genotype association analysis and allele effect calculations were performed using the TASSEL software. Two models, the general linear model (GLM) adjusted using the O-matrix (GLM [Q]) and the mixed linear model (MLM) correcting for both Q-matrix and K-matrix (MLM $[\mathrm{Q}+\mathrm{K}]$ ), were used to reduce errors from population structure and relative kinship. Those with $P<0.01$ adjusted by the Bonferroni method were defined as significant trait-associated SNPs.

\section{Validation of favorable SNPs}

The $F_{1}$ hybrid progeny population described above, consisting of 241 lines, was used for validation of the favorable SNPs via a genomic DNA PCR strategy with special primers (Additional file 2) designed based on SNP sequence according to the method described by Chen et al. [37]. DNA samples were extracted from young leaves of each line using a DNeasy plant mini kit (Tiangen, Beijing, China). PCR amplification was performed in a $20 \mathrm{~mL}$ reaction volume consisting of $1 \times$ EasyTaq buffer, $0.2 \mathrm{mmol} / \mathrm{L}$ dNTPs, $0.5 \mathrm{mmol} / \mathrm{L}$ of each of the forward and reverse 
primers, 2.5 units of EasyTaq DNA Polymerase (Transgen Biotech, China), $100 \mathrm{ng}$ of DNA template, and an appropriate amount of sterile double-distilled water. The amplification schedule was run as follows: an initial denaturation at $95^{\circ}$ for $5 \mathrm{~min}$, followed by 33 cycles of $95^{\circ}$ for $30 \mathrm{~s}, 58^{\circ}$ for $45 \mathrm{~s}$, and $72^{\circ}$ for $1 \mathrm{~min}$. PCR products were separated using $8 \%$ polyacrylamide gels, and silver staining was conducted according to the method of Luan et al. [26]. Molecular weights were estimated using a DNA marker (DNA Marker 2000, BioTeke Co., Beijing, China). Clear amplified bands were recorded as 1 and the absence of bands was recorded as 0 . The products of clean bands were sequenced at Biomarker Technologies Corporation to ensure the authenticity of the target sequences.

Spearman correlation analysis using IBM SPSS Statistics 19.0 (IBM, NY, USA) was performed to determine if the ramet number in the $\mathrm{F}_{1}$ hybrid progeny had any correlation with the SNPs. A difference between means was considered statistically significant at $P<0.05$.

\section{Identification of candidate genes and quantitative PCR (qPCR) analysis}

Sequences of favorable SNPs were used to blast the ramie genome [38], and genes located in the region $150 \mathrm{~kb}$ upstream or downstream of the ramet number associated SNPs were identified as candidate associated genes.

Three replicates of each of two germplasm plants (Chuanzhu NO.2 and Quxianzhuma, Additional file 1) were used for the qPCR analysis. For the expression of candidate genes, total RNA was extracted from the leaf and stem bark of each germplasm using a plant RNA purification kit (Tiangen, Beijing, China). Briefly, $0.5 \mu \mathrm{g}$ total RNA was used to synthesize cDNA (in $10-\mathrm{mL}$ reaction volumes) using a PrimeScript RT perfect real-time reagent kit (TaKaRa, Japan). Subsequently, the cDNA was diluted four times. The qPCR analysis was performed using a Lightcycler 480 engine (Roche, Germany) using the $2 \times$ T5 Fast qPCR Mix (SYBR Green I, TSINGKE Biological Technology, Beijing, China) in a $20-\mathrm{mL}$ reaction volume consisting of $10 \mu \mathrm{L} 2 \times$ T5 Fast qPCR mix, $0.4 \mu \mathrm{mol} / \mathrm{L}$ of each of the forward and reverse primers (listed in Additional file 3), $2 \mu \mathrm{L}$ diluted cDNA, and an appropriate amount of sterile double-distilled water. The PCR conditions consisted of an initial denaturation step at $95^{\circ}$ for $1 \mathrm{~min}$, followed by 40 cycles of denaturation at $95^{\circ}$ for $10 \mathrm{~s}$, annealing at $60^{\circ}$ for $5 \mathrm{~s}$, and extension at $72^{\circ}$ for $15 \mathrm{~s}$. The relative expression was calculated using the $2^{-\Delta \Delta \mathrm{Ct}}$ method [39] using the housekeeping gene $18 S$ as an internal control.

\section{Results}

Phenotypic characteristics of ramet number

For the 112 core germplasms, the ramet number of each germplasm varied widely in each season (Table 1), ranging from 2.00 to 13.83, with an average of 7.66, in May 2016; from 5.00 to 18.00 , with an average of 9.48 , in August 2016 ; and from 4.33 to 21.50 , with an average of 11.68 , in November 2016. These data illustrated the large variation amplitude of the core germplasm population, indicating that it could be an excellent population for marker-trait GWAS

For the hybrid progeny population (validation population), the ramet number of 241 lines was analyzed in two seasons (in March and November 2017). The variation in ramet number was 10-fold in March 2017, from 1 to 10, and 9-fold in November 2017, from 2 to 18 (Table 1). The variation of the ramet number in the two seasons showed a normal distribution curve (Additional file 4). The large variation in ramet number in the validation population was an advantage for validation of favorable SNPs.

\section{SNP-based genotyping of ramie accessions}

To finely map the ramet number associated genes and investigate beneficial haplotypes in the ramie germplasm, a haplotype map of the 112 core germplasms was constructed using the SLAF-seq approach. In total, over $364.29 \mathrm{Mb}$ reads were generated for the 112 genotypes (Table 2). Approximately 2,458,923 high-quality SLAF tags were identified from the total reads, and 336,623 of the high-quality SLAF tags showed high polymorphism. The SLAF tags used to call the SNPs had an average depth of 10.89-fold per individual among the 112 germplasms. In total, 1,113,711 SNPs were initially called for this set of lines; after further exclusion at MAF $>0.05$ and integrity $>0.8,108,888$ high-consistency SNPs were retained for the analysis. These SNPs were used to assess population structure and GWAS analysis.

\section{Population structure and kinship}

Considering the fact that the authenticity of QTL mapping could be affected by population structure, it is critical to understand the structure matrix in GWAS populations. In this study, the number of subgroups in the 112 core germplasms was estimated with two methods based on the genotypic database. First, the Bayesian clustering from $K=1$ to 10 was calculated with the STRUCTURE software. The delta $K$ value reached the lowest point at $K=4$ (Fig. 1a), suggesting that the population could be divided into four subgroups (Fig. 1b, Additional file 1): Groups I, II, III, and IV. Secondly, an NJ phylogeny based on genetic distances also showed that the 112 core germplasms were outlined with four main clusters (Fig. 1c), which was consistent with the result of the STRUCTURE analysis, despite some accessions overlapping in the four clusters. 
Table 1 Phenotypic variation of ramet number in association analysis and validation population

\begin{tabular}{lllllll}
\hline Population & Detected stage & Mean & SD & Min & Max & CV (\%) \\
\hline Association analysis population & May, 2016 & 7.66 & 2.34 & 2.00 & 13.83 & 30.49 \\
& August, 2016 & 9.48 & 2.61 & 5.00 & 18.00 & 27.56 \\
& November, 2016 & 11.68 & 3.15 & 4.33 & 21.50 & 26.97 \\
Validation population & March, 2017 & 5.29 & 1.96 & 1.00 & 10.00 & 37.14 \\
& November, 2017 & 8.29 & 3.23 & 2.00 & 18.00 \\
\hline
\end{tabular}

$S D$ standard deviation, $C V$ coefficient of variation

\section{Marker-trait GWAS}

The GWAS was performed using GLM and MLM models with 108,888 high-consistency SNPs. To confirm the potentially significant SNPs associated with ramet number, genotypes from three stages of the 112 core germplasms were analyzed. For the GLM model, a total of 2553, 2256, and 4288 significant SNPs were detected at $P \leq 0.01$ in May, August, and November in 2016, respectively, and 44 common significant SNPs were shown in all three stages (Additional files 5 and 6). For the MLM model, 1067, 815, and 4049 significant SNPs were found at $P \leq 0.01$ in the three stages, and four of the SNPs (Marker38532-124, Marker54845-111, Marker57363-43, and Marker75993-18) emerged in all three stages at the same time. Some of the detected significant SNPs emerged at least twice in the same stages; for example, the SNP (Marker20170-64) generated using the MLM model emerged twice in May.

\section{Identification and validation of favorable SNPs associated with ramet number}

To understand the effects of allelic variation on the ramet number, 20 significant SNPs, which emerged at least twice in the three stages analyzed, were identified as favorable alleles. An $F_{1}$ hybrid progeny population of 241 lines and its parents were used to verify the authenticity of the favorable SNPs using genomic DNA PCR with specific primers (Additional file 2). First, DNA of the parents was used as a template for amplification with the 20 pairs of primers, and the results showed that two (Marker20170-64 and Marker142939-43) of the favorable SNPs amplified a product in one but not in the other parent (Additional file 7). Five of the favorable SNPs (Marker70439-41, Marker13742-63, Marker15847-131,

Table 2 Summary of statistic data generated by specific-locus amplified fragment sequencing (SLAF-seq) technology

\begin{tabular}{ll}
\hline Name & Number \\
\hline Total reads & $364.29 \mathrm{Mb}$ \\
High-quality SLAF tags & $2,458,923$ \\
High polymorphism reads & 336,623 \\
High-consistency SNPS & 108,888
\end{tabular}

SNP single nucleotide polymorphisms
Marker21174-109, and Marker18389-39) amplified a product in both parents. The remaining 13 favorable SNPs did not amplify any product in either parent. After verifying the amplification products by sequencing, Marker20170-64 and Marker142939-43 SNPs were selected for further amplification using the DNA samples of the 241 lines as templates. As shown in Fig. 2, amplification was successful in 126 of the 241 lines using Marker20170-64, which was close to the ratio of 1:1 (Table 3), whereas 222 of the 241 lines were amplified using Marker142939-43. The results of the correlation analysis between the trait (ramet numbers of the 241 lines) and the amplification results, suggests that the SNP Marker20170-64 was associated with the ramet number in ramie (Table 4).

\section{Candidate gene identification and expression analysis}

As validated above, the SNP Marker20170-64 may be a major genetic locus responsible for ramet number in ramie. Thus, the haplotype block structure was investigated within $150 \mathrm{~kb}$ on either side of Marker20170-64 to determine candidate genes. A total of nine genes were found in the region of Marker20170-64. Bioinformatic analysis showed that three of these genes lacked any definite annotation for their biological functions and four genes were linked to biological pathways involved in plant growth and development (Additional file 3).

To determine the genes responsible for ramet number in ramie, a qPCR analysis was performed using two ramie varieties with significantly different ramet numbers (Fig. 3a). The results showed that six genes (Bn23049, Bn23037, $B n 23055, B n 23053, B n 23057$, and Bn23041) were upregulated in different tissues (leaf and stem bark) of the high ramet number genotype Quxianzhuma (Fig. 3b). Two (Bn23037 and Bn23053) of these six genes showed functions in regulating root development, one (Bn23049) in regulating seed germination, and one (Bn23041) was associated with cell fate determination and maintenance of floral/inflorescence/shoot apical meristem identity.

\section{Discussion}

Application of GWAS in ramie

Ramet number is a significant quantitative trait in ramie, which is controlled by multigenes. Understanding the 


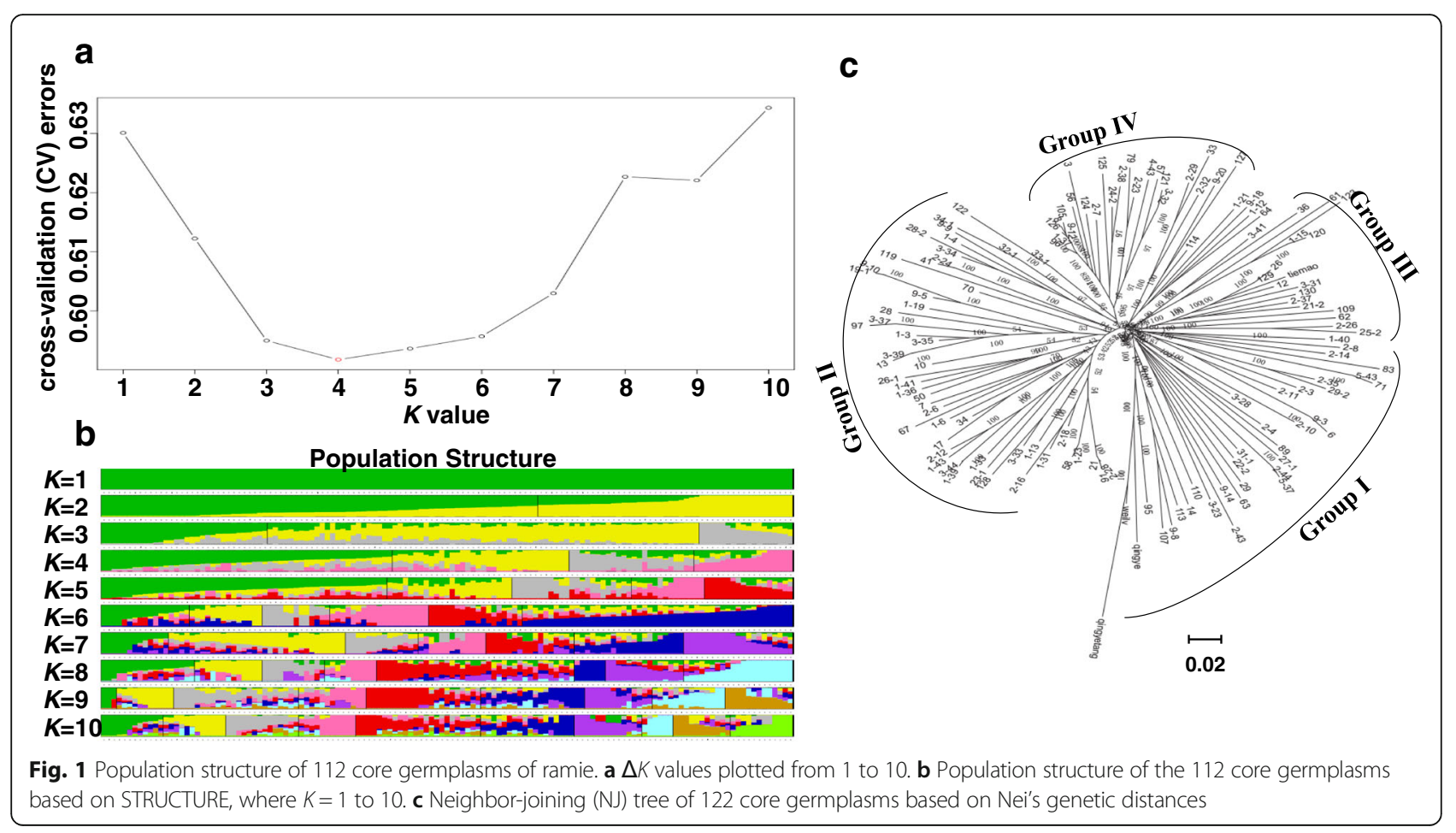

mechanism of ramet number determination through gene/SNP/QTL analysis and breeding high ramet number cultivars using marker-assisted selection are thought to be the most practical and effective strategies to manage this trait. However, it is difficult to reliably identify effective SNPs/QTLs associated with ramet number in ramie for various reasons, including the low genome coverage of the available molecular markers, the slight effects of most loci, the limited allelic segregation, and recombination of biparental populations in linkage mapping. In this study, we used an association population consisting of 112 core germplasms collected from China, Japan, India, Indonesia, and Cuba, for ramet number-associated SNP detection, which offered more historical recombination events, to overcome the limitations of biparental populations.

In this study, GWAS, which has been used to map complex quantitative traits in plants [21, 40-42], was performed for the first time in ramie to detect association between SNPs and quantitative traits. The power of GWAS depends on four main factors: the richness of genetic diversity, the veracity of trait acquisition, the marker density, and the statistical methods. The core germplasms used for the GWAS in our study were collected from different regions of China, representing the typical characteristics of various ramie varieties. Furthermore, they have high levels of genotypic and phenotypic diversity (Table 1), which is suitable for GWAS. Because ramet production in ramie can be affected by nutrient, harvest time, and other environmental factors [43, 44], phenotypic data from three stages were analyzed to ensure that the detected SNPs were reliable. Two models, GLM and MLM, were employed to reduce errors associated with population structure and relative kinship. Each step was verified to ensure that the significant SNPs identified in this study were reliable and reproducible.

\section{Verification of significant SNPs associated with ramet number}

Some false positives may occur in plant GWASs because of genetic heterogeneity among different varieties, resulting in some SNPs being significant in one population but not in others [45]. Therefore, it is necessary to verify the significant SNPs detected using GWAS with different populations. In this study, 20 significant SNPs, which emerged at least twice in the three stages analyzed were identified as favorable alleles and verified using an $\mathrm{F}_{1}$ hybrid progeny population of 241 lines. The SNP (Marker20170-64) was found to be associated with ramet number in the hybrid progeny population, confirming its authenticity and demonstrating the need to verify significant SNPs detected by GWAS. Additionally, three loci (Marker20170-57, Marker20170-1, Marker 20,170-103) close to the Marker20170-64 were identified using SLAF-seq technology. All of these loci emerged at least twice in the three stages analyzed (Additional file 8), further confirming the authenticity of the Marker20170-64.

\section{Identification of candidate genes}

In perennial plants such as ramie, Potentilla anserina, Rubus saxatilis, Linnaea borealis, and tussock, ramet number is a complex trait [46-48]. For instance, the size 


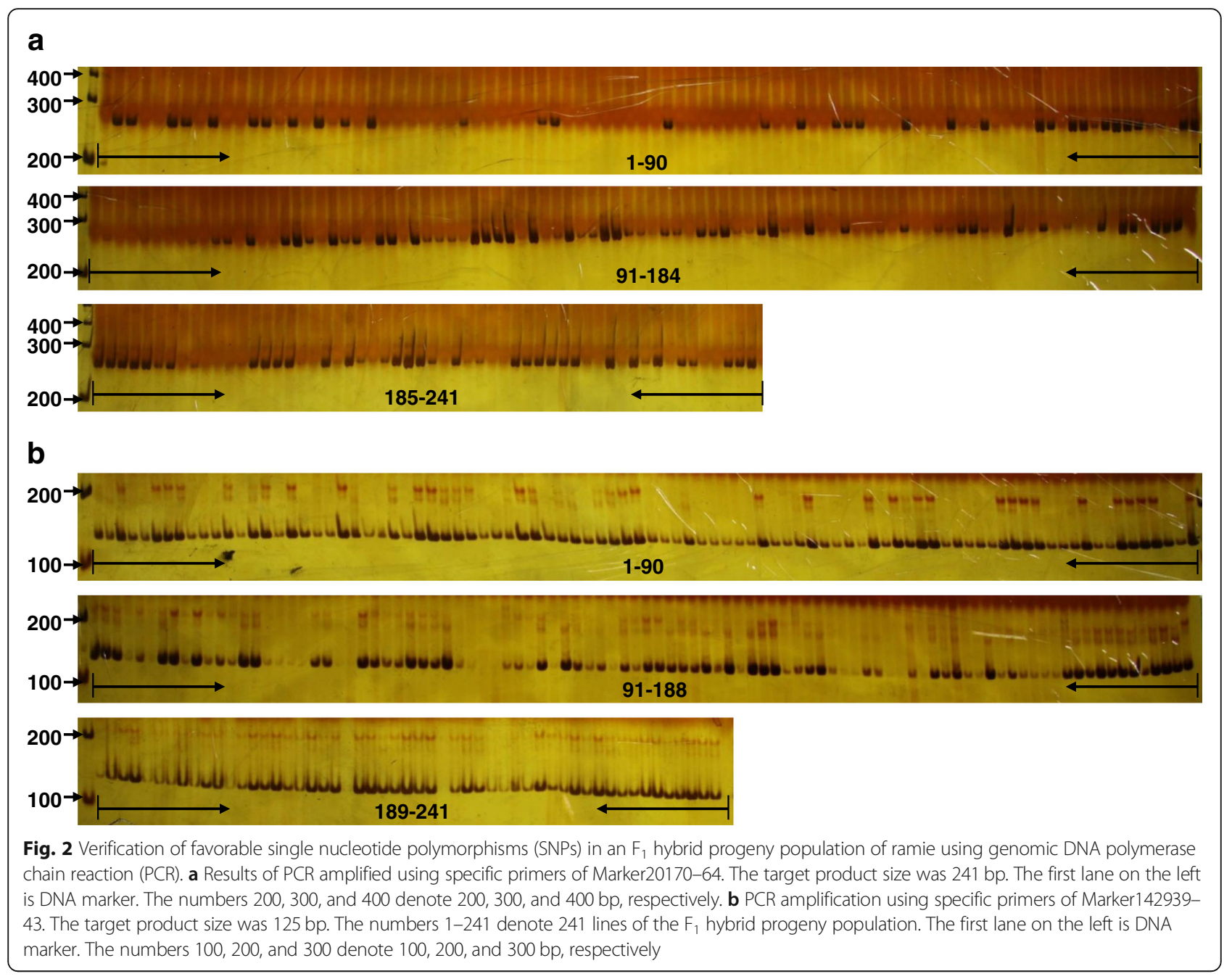

of the ramet population in Carex humilis increases with age; flowering ramets do not produce any offspring ramets, and larger parent ramets produce more and larger offspring ramets [47]. At the molecular level, many genes that control tiller number per plant have been cloned from diverse plant species. PvSPL2, an SBP-box transcription factor that affects lignin biosynthesis in switchgrass, predominantly modulates tiller initiation and stem elongation [49]. In rice, OsHTD2, which is involved in the strigolactone biosynthetic pathway, negatively regulated tiller bud outgrowth via the strigolactone

Table 3 Test for goodness-of-fit $\left(X^{2}\right)$ between ramet number and $P C R$ results in validation population

\begin{tabular}{lllll}
\hline $\begin{array}{l}\text { Amplification } \\
\text { band type }\end{array}$ & $\begin{array}{l}\text { Ramet } \\
\text { number }(O)\end{array}$ & $\begin{array}{l}\text { Ramet } \\
\text { number }(E)\end{array}$ & $O-E$ & $(|O-E|-1 / 2)^{2} / E$ \\
\hline None & 115 & 120.5 & -5.5 & 0.2075 \\
Exist & 126 & 120.5 & 5.5 & 0.2075 \\
Total & 241 & 241 & 0 & 0.4150 \\
\hline
\end{tabular}

$O$ denotes observed value, $E$ denotes theoretical value pathway [50]. OsIAA6, a member of the rice $A u x / I A A$ gene family, is involved in drought tolerance and tiller outgrowth [51]. These research findings show that there are multiple factors and pathways controlling ramet/ tiller number in different plants. In this study, the PCR results in the validation population showed that the ratio of presence and absence of bands in the offspring with high and low ramet number was close to 1:1. These results indicate that ramie ramet number may be controlled by a single gene pair. However, the average ramet number of the validation population was normally distributed, which denotes that the ratio of low ramet number to high ramet number was not 1:1, indicating that ramie ramet number is a quantitative trait controlled by

Table 4 The correlation ( $r$ ) between single nucleotide polymorphisms (SNPs) and ramet number

\begin{tabular}{lll}
\hline Trait & Marker20170 & Marker142939 \\
\hline Ramet number in March 2017 & $-0.201^{a}$ & 0.039 \\
Ramet number in November 2017 & -0.094 & 0.013
\end{tabular}

adenotes significant difference between phenotypes and genotypes at $P<0.01$ 
a

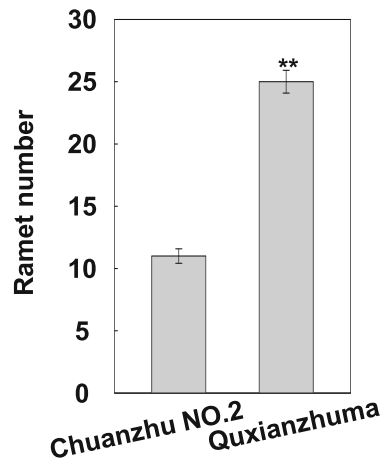

b

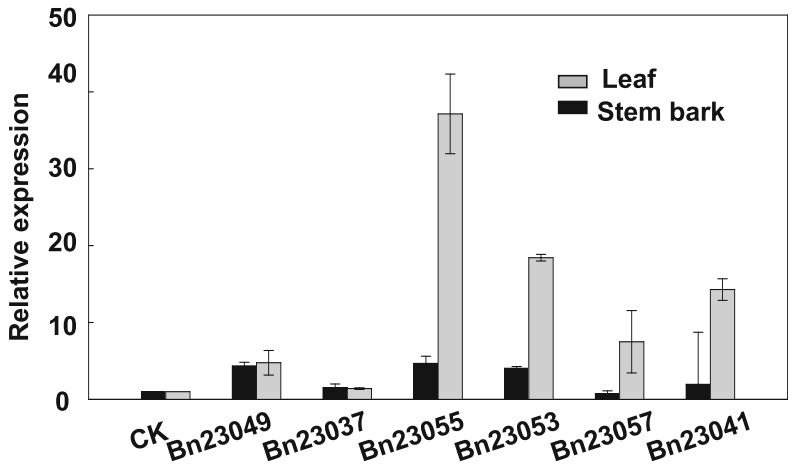

Fig. 3 a Ramet number of two ramie varieties (Chuanzhu NO.2 and Quxianzhuma). ${ }^{* *}$ denotes statistically significant differences from the control at $P<0.01$. b Expression of candidate genes (Bn23049, Bn23037, Bn23055, Bn23053, Bn23057, and Bn23041) in leaf and stem bark of Chuanzhu NO.2 and Quxianzhuma. The $2^{-\Delta \Delta C t}$ method was used to calculate the relative expression level of target genes. The expression in Chuanzhu NO.2 was used as control (CK) and its value was set as 1. Data are means \pm SE of three replicates. Each replicate consisted of material from three plants that grew to $40-50 \mathrm{~cm}$ in height

multiple genes. By comparing the Marker20170-64 to the ramie genome, we identified six genes that appeared to be related to ramet number, suggesting that ramet number is controlled by multiple genes in ramie.

The potential role of these six target genes in controlling ramet number was indicated by their up-regulated expression in the high ramet number genotype Quxianzhuma and their roles in the regulation of root development (Bn23037 and Bn23053), seed germination (Bn23049), and cell fate determination and maintenance of floral/inflorescence/shoot apical meristem identity (Bn23041). A homologous gene of Bn23037 in Arabidopsis, AtERF4 (ERF, ethylene response factor), has a vital function in regulating leaf growth and development by responding to nutrition stress [52]. AtTZF5, whose CDS shows the highest homology with $B n 23049$, affects seed germination by controlling genes critical for abscisic acid and gibberellic acid response [53]. AtRING1a, a homologous gene of Bn23041, plays a primary role in the maintenance of meristem function by inhibiting the expression of a Class I KNOTTEDlike homeobox transcription factor [54]. Plant hormones such as ethylene, abscisic acid, and gibberellic acid play a key role in controlling multiple aspects of plant growth and development, including tissue differentiation, root elongation, shoot branching, and flowering time [55]. The up-regulated expression of the six candidate genes may affect the changes of plant hormones and further regulate ramet development in ramie. However, because of the dearth of related literature on the ramet number of ramie, the biological function of the candidate genes identified in this study should be further verified using biological experiments. Taken together, the genes identified in our study could be used as candidate resources for the molecular improvement of ramet number in ramie.

\section{Conclusions}

Using the GWAS method for the first time in ramie, we genotyped 112 ramie core germplasms and identified 44 SNPs significantly associated with ramet number across three stages and nine genes. Collectively, the identified SNPs and genes could be used as candidate resources for the molecular improvement of ramet number in ramie. In conclusion, our study provides technological strategies for quantitative trait studies of ramie.

\section{Additional files}

Additional file 1: One hundred twelve core germplasms used for SNP genotyping. (XLSX $14 \mathrm{~kb}$ )

Additional file 2: $\mathrm{qPCR}$ primer sequences of favorable SNPS and reference gene. (XLSX $11 \mathrm{~kb}$ )

Additional file 3: Primers and functional description of candidate genes. (XLSX $11 \mathrm{~kb}$ )

Additional file 4: Normal distribution curve of the ramet number in hybrid progeny population in (a) dMarch 2017 and (b) November 2017. (PDF $20 \mathrm{~kb}$ )

Additional file 5: SNPS generated from GLM and MLM analysis when $P<0.01$. (XLSX $364 \mathrm{~kb}$ )

Additional file 6: Common significant SNPs in GLM analysis and MLM analysis. (XLSX $11 \mathrm{~kb}$ )

Additional file 7: Results of polymerase chain reaction (PCR) amplification using specific primers of significant single nucleotide polymorphisms (SNPs) in parents of the $F_{1}$ hybrid progeny population. M denotes $100 \mathrm{bp}$ DNA Ladder, the bands from bottom to the top represent 100, 200, 300, $400,500,600,700,800,900,1000$, and 1500 bp, respectively. The numbers 1-20 represent Marker70439-41, Marker13742-63, Marker29152-60, Marker42663-35, Marker59771-76, Marker142939-43, Marker15847-131, Marker121162-120, Marker20170-64, Marker21174-109, Marker130175-63, Marker18389-39, Marker12702-125, Marker178103-63, Marker21112-51, Marker24814-129, Marker31623-105, Marker39027-122, Marker73630-112, and Marker38532-124, respectively. For each SNP marker, amplifications from left to right represent three female (Hejiangqingma) and two male (Zhongzhu NO.1) parents. (PDF $45 \mathrm{~kb}$ ) 
Additional file 8: Additional SNP loci near the Marker20170-64. (XLSX $10 \mathrm{~kb})$

\section{Abbreviations}

GLM: General linear model; GWAS: Genome-wide association study; MLM: Mixed linear model; NJ: Neighbor-joining; PCR: Polymerase chain reaction; qPCR: quantitative PCR; QTL: Quantitative trait loci; SLAF-seq: Specificlocus amplified fragment sequencing; SNPs: Single nucleotide polymorphisms

\section{Acknowledgments}

We sincerely thank Editage (www.editage.cn) for English language editing.

\section{Funding}

This work was financially supported by grants from the National Natural Science Foundation of China (31801413), the Science and Technology Innovation Project of the Chinese Academy of Agricultural Sciences (CAAS-ASTIP-2016-IBFC04) and the Ramie Breeding Station of the National Fiber Industry Technology System (CARS-19-E02). The funding bodies had no role in the design of the study, the collection, analysis, and interpretation of data and in writing the manuscript

\section{Availability of data and materials}

All data presented in this study are provided either in this published article or its additional files. The NCBI SRA accession numbers for the SLAF-seq reads was SRP159744.

\section{Authors' contributions}

KMC and MBL performed most of the experiments. KYH, PC, JKC, and GG helped in phenotypic data collection. AGZ and CMY designed the experiments with assistance from HPX. All authors read and approved the final manuscript.

\section{Ethics approval and consent to participate}

Not applicable.

\section{Consent for publication}

Not applicable.

\section{Competing interests}

The authors declare that they have no competing interests.

\section{Publisher's Note}

Springer Nature remains neutral with regard to jurisdictional claims in published maps and institutional affiliations.

Received: 22 April 2018 Accepted: 26 November 2018

Published online: 12 December 2018

\section{References}

1. Lee J, Kim AR, Lee JJ. Ramie leaf extracts suppresses adipogenic differentiation in 3T3-L1 cells and pig preadipocytes. Asian Australas J Anim Sci. 2016; 29:1338-44.

2. Zhou JH, Yang QW, Lan CY, Ye ZH. Heavy metal uptake and extraction potential of two Bechmeria nivea (L.) gaud. (ramie) varieties associated with chemical reagents. Water Air Soil Poll. 2010;211:359-66.

3. Lei M, Yue QL, Chen TB, Huang ZC, Liao XY, Liu YR, Zheng GD, Chang OR. Heavy metal concentrations in soils and plant around Shizuyuan mining area of Hunan provice. Acta Ecol Sin. 2005:25(5):1146-51 (in Chinese).

4. Yang B, Zhou M, Shu WS, Lan CY, Ye ZH, Qiu RL, Jie YC, Cui GX, Wong MH. Constitutional tolerance to heavy metals of a fiber crop, ramie (Boehmeria nivea), and its potential usage. Environ Poll. 2010;158:551-8.

5. Kariali E, Sarangi S, Panigrahi R, Panda BB, Mohapatra PK. Variation in senescence pattern of different classes of rice tillers and its effect on panicle biomass growth and grain yield. Am J Plant Sci. 2012;3:1047-57.

6. Miller BC, Hill JE, Roberts SR. Plant population effects on growth and yield in water-seedd rice. Agron J. 1991;83:291-7.

7. Gravois KA, Helms RS. Path analysis of rice yield components as affected by seeding rate. Agron J. 1992;84:1-4.

8. Zheng YL, Feng YL, Liu WX, Liao ZY. Growth, biomass allocation, morphology, and photosynthesis of invasive Eupatorium adenophorum and its native congeners grown at four irradiances. Plant Ecol. 2009; 203:263-71.

9. Chen KM, Xiong HP, Zhu AG, Luan MB, Hou M, Chen P, Chen JK, Gao $\mathrm{G}, \mathrm{Yu}$ CM. Correlation and regression analysis of biomass and biomass components of ramie. Plant Fiber Sci in China. 2017;39(6):289-91 (in Chinese).

10. Bai YC, Wang H, Guo T, Li LL, Yang RF, She W, Cui GX. Effect of root rot on fiber yield and yield-related traits of ramie (Boehmeria nivea). Acta Prataculturae Sin. 2017;26(9):45-56 (in Chinese).

11. Han YP, Zhao X, Liu DY, L YH, Lightfoot DA, Yang ZJ, Zhao L, Zhou G, Wang ZK, Huang L, Zhang ZW, Qiu LJ, Zheng HK, Li WB. Domestication footprints anchor genomic regions of agronomic importance in soybeans. New Phytol. 2016;209:871-84

12. Sun XW, Liu DY, Zhang XF, Li WB, Liu H, Hong WG, Jiang CB, Guan N, Ma CX, Zeng HP, Xu CH, Song J, Huang L, Wang CM, Shi JJ, Wang R, Zheng XH, Lu CY, Wang XW, Zheng HK. SLAF-seq: an efficient method of large scale de novo SNP discovery and genotyping using highthroughput sequencing. PLoS One. 2013:8:e58700.

13. Li CC. Diallel analysis of yield and its component traits in rice (Oryza sativa L.) J Agr Assoc China. 1975:47:41-56.

14. Xiao YJ, Tong H, Yang XH, Xu SZ, Pan QC, Qiao F, et al. Genome-wide dissection of the maize ear genetic architecture using multiple populations. New Phytol. 2016;210:1095-106.

15. Li H, Peng ZY, Yang XH, Wang WD, Fu JJ, Wang JH, et al. Genome-wide association study dissects the genetic architecture of oil biosynthesis in maize kernels. Nat Genet. 2013;45(1):43-50.

16. Huang $X H$, Wei $X H$, Sang $T$, Zhao Q, Feng Q, Zhao Y, et al. Genome-wide association studies of 14 agronomic traits in rice landraces. Nat Genet. 2010; 42:961-7.

17. Zhao YL, Wang HM, Chen W, Li YH. Genetic structure, linkage disequilibrium and association mapping of verticillium wilt resistance in elite cotton (Gossypium hirsutum L.) germplasm population. PLoS One. 2014:9:e86308.

18. Liu HJ, Luo X, Niu LY, Xiao YJ, Chen L, Liu J, Wang XQ, Jin ML, Li WQ, Zhang $\mathrm{QH}$, Yan JB. Distant eQTLs and non-coding sequences play critical roles in regulating gene expression and quantitative trait variation in maize. Mol Plant. 2016:10:414-26.

19. Morris GP, Ramu P, Deshpande SP, Hash CT, Shah T, Upadhyaya HD, RieraLizarazu O, Brown PJ, Acharya CB, Mitchell SE, Harriman J, Glaubitz JC, Buckler ES, Kresovich S. Population genomic and genome-wide association studies of agroclimatic traits in sorghum. Proc Natl Acad Sci U S A. 2013;110:453-8.

20. Jia GQ, Huang XH, Zhi H, Zhao Y, Zhao Q, Li WJ, et al. A haplotype map of genomic variations and genome-wide association studies of agronomic traits in foxtail millet (Setaria Italica). Nat Genet. 2013:45:957-61.

21. Atwell S, Huang YS, Vilhjalmsson BJ, Willems G, Horton M, Li Y, et al. Genome-wide association study of 107 phenotypes in Arabidopsis thaliana inbred lines. Nature. 2010;465(7298):627-31.

22. Li TG, Ma XF, Li NY, Zhou L, Liu Z, Han HY, Gui YJ, Bao YM, Chen JY, Dai XF. Genome-wide association study discovered candidate genes of Verticillium wilt resistance in upland cotton (Gossypium hirsutum L.). Plant Biotechnol J. 2017; 15:1520-32.

23. Li K, Wang HW, Hu XJ, Liu ZF, Wu YJ, Huang CL. Genome-wide association study reveals the genetic basis of stalk cell wall components in maize. PLoS One. 2016:11:e0158906.

24. Chao DY, Chen Y, Chen JG, Shi SL, Chen ZR, Wang CC, Danku JM, Zhao FJ, Salt DE. Genome-wide association mapping identifies a new arsenate reductase enzyme critical for limiting arsenic accumulation in plants. PLoS Biol. 2014;12:e1002009.

25. Liu TM, Tang SW, Zhu SY, Tang QM. QTL mapping for fiber yield-related traits by constructing the first genetic linkage map in ramie (Boehmeria nivea L. gaud). Mol Breed. 2014;34:883-92.

26. Luan MB, Liu CC, Wang XF, Xu Y, Sun ZM, Chen JH. SSR markers associated with fiber yield traits in ramie (Boehmeria nivea L. Gaudich). Ind Crop Prod. 2017;107:439-45.

27. Zhou QH, Zhou C, Zheng W, Mason AS, Fan SY, Wu CJ, Fu DH, Huang YJ. Genome-wide SNP markers based on SLAF-Seq uncover breeding traces in rapeseed (Brassica napus L.). Front Plant Sci. 2017:8:648.

28. Li H, Durbin R. Fast and accurate short read alignment with burrows-wheeler transform. Bioinformatics. 2009:25:1754-60.

29. McKenna A, Hanna M, Banks E. The genome analysis toolkit: a mapreduce framework for analyzing next-generation DNA sequencing data. Genome Res. 2010;20:1297-303. 
30. Li H, Handsaker B, Wysoker A, Fennell T, Ruan J, Homer N, Marth G, Abecasis $\mathrm{G}$, Durbin R. The sequence alignment map format and SAMtools. Bioinformatics. 2009;25(16):2078-9.

31. Alexander DH, Novembre J, Lange K. Fast model-based estimation of ancestry in unrelated individuals. Genome Res. 2009;19:1655-64.

32. Earl DA, Vonholdt BM. STRUCTURE HARVESTER: a website and program for visualizing STRUCTURE output and implementing the Evanno method. Conserv Genet Resour. 2011:4:359-61.

33. Mezmouk S, Dubreuil P, Bosio M, Decousset L, Charcosset A. Effect of population structure corrections on the results of association mapping tests in complex maize diversity panels. Theor Appl Genet. 2011;122: 1149-60.

34. Jakobsson M, Rosenberg NA. CLUMPP: a cluster matching and permutation program for dealing with label switching and multimodality in analysis of population structure. Bioinformatics. 2007:23:1801-6.

35. Saitou N, Nei M. The neighbor-joining method: a new method for reconstructing phylogenetic trees. Mol Biol Evol. 1987:4:406-25.

36. Hardy OJ, Vekemans X. SPAGEDi: a versatile computer program to analyse spatial genetic structure at the individual or population levels. Mol Ecol Notes. 2002;2:618-20.

37. Chen W, Yao JB, Chu L, Li Y, Guo XM, Zhang YS. The development of specific SNP markers for chromosome 14 in cotton using next-generation sequencing. Plant Breed. 2014;133:256-61.

38. Luan MB, Jian JB, Chen P, Chen JH, Chen JH, Gao Q, Gao G, Zhou JH, Chen KM, Guang XM, Chen JK, Zhang QQ, Wang XF, Fang L, Sun ZM, Bai MZ, Fang XD, Zhao SC, Xiong HP, Yu CM, Zhu AG. Draft genome sequence of ramie, Boehmeria nivea (L.) Gaudich. Mol Ecol Resour. 2018; 18(3):639-45.

39. Livak KJ, Schmittgen TD. Analysis of relative gene expression data using real-time quantitative PCR and the $2^{-\Delta \Delta C T}$ method. Methods. 2001;25:402e408.

40. Ingvarsson PK, Street NR. Association genetics of complex traits in plants. New Phytol. 2011;189:909-22

41. Lu Q, Zhang M, Niu X, Wang S, Xu Q, Feng Y, Wang CH, Deng HZ, Yuan XP, Yu HY, Wang YP, Wei XH. Genetic variation and association mapping for 12 agronomic traits in indica rice. BMC Genomics. 2015; 16:1067.

42. Crowell S, Korniliev P, Falcao A, Ismail A, Gregorio G, Mezey J, McMouch S. Genome-wide association and high-resolution phenotyping link Oryza sativa panicle traits to numerous trait-specific QTL clusters. Nat Commun. 2016;7:10527.

43. Dai XB, Li LJ, Peng DX. Effect of nitrogen and potassium fertilizer on ramie plant height and effective ramet rate. Hubei Plant Prot. 2014;5: 19-22 (in Chinese).

44. Xiao ZP. Study of high yield and quality cultivation techniques of ramie in hilly area. Hunan Agric Sci. 1997;2:21-4 (in Chinese).

45. Myles S, Dan D, Barrett J, Stoneking M, Timpson N. Worldwide population differentiation at disease-associated SNPs. BMC Med Genet. 2008;1:1-10.

46. Eriksson O. Stochastic population dynamics of clonal plants: numerical experiments with ramet and genet models. Ecol Res. 1994;9:257-68.

47. Sofie W. Carex humilis-a caespitose clonal plant: ramet demography, ring formation, and community interactions. Sweden: Lund University Publications; 2002.http://lup.lub.lu.se/record/464568.

48. Münzbergová Z. Kr ivánek M, Bucharová a, Juklíc Ková V, Herben T. Ramet performance in two tussock plants - do the tussock-level parameters matter? Flora. 2005;200:275-84.

49. Wu ZY, Cao YP, Yang RJ, Qi TX, Hang YQ, Lin H, Zhou G, Wang ZY, Fu CX. Switchgrass SBP-box transcription factors PVSPL1 and 2 function redundantly to initiate side tillers and affect biomass yield of energy crop. Biotechnol Biofuels. 2016;9:3-15.

50. Liu WZ, Wu C, Fu YP, Hu GC, Si HM, Zhu L, Luan WJ, He ZQ, Sun ZX. Identification and characterization of HTD2: a novel gene negatively regulating tiller bud outgrowth in rice. Planta. 2009;230:649-58.

51. Jung $H$, Lee DK, Choi YD, Kim JK. OsIAA6, a member of the rice Aux/IAA gene family, is involved in drought tolerance and tiller outgrowth. Plant Sci. 2015:236:304-12

52. Liu W, Karemera NJU, Wu T, Yang YF, Zhang XZ, Xu XF, Wang Y, Han ZH. The ethylene response factor AtERF4 negatively regulates the iron deficiency response in Arabidopsis thaliana. PLoS One. 2017;12(10):e0186580.

53. Bogamuwa S, Jang J. The Arabidopsis tandem $\mathrm{CCCH}$ zinc finger proteins AtTZF4, 5 and 6 are involved in light-, abscisic acid- and gibberellic acid-mediated regulation of seed germination. Plant Cell Environ. 2013; 36:1507-19.

54. Lin X, Shen WH. Polycomb silencing of KNOX genes confines shoot stem cell niches in Arabidopsis. Curr Biol. 2008;18:1966-71.

55. Santner A, Estelle M. Recent advances and emerging trends in plant hormone signaling. Nature. 2009;459(7250):1071-8.
Ready to submit your research? Choose BMC and benefit from:

- fast, convenient online submission

- thorough peer review by experienced researchers in your field

- rapid publication on acceptance

- support for research data, including large and complex data types

- gold Open Access which fosters wider collaboration and increased citations

- maximum visibility for your research: over $100 \mathrm{M}$ website views per year

At $\mathrm{BMC}$, research is always in progress.

Learn more biomedcentral.com/submissions 